\title{
Psychological factors associated with smoking and quitting: addiction map of Turkey study
}

This article was published in the following Dove Press journal:

Neuropsychiatric Disease and Treatment

\author{
Hüseyin Ünübol' \\ Gökben Hızlı Sayar ${ }^{2}$ \\ 'Üsküdar University, Institute of Social \\ Sciences, Department of Applied \\ Psychology, Istanbul, Turkey; ${ }^{2}$ Üsküdar \\ University, Institute of Social Sciences, \\ Department of Clinical Psychology, \\ Istanbul, Turkey
}

Background: Smoking is the most important modifiable factor in increased morbidity and premature mortality. This study aims to examine the psychological factors associated with smoking and quitting in a broad, nationally representative sample.

Participants and methods: The sample included a total of 24.494 adult individuals. Participants were divided into three groups as smokers, non-smokers, and "ex-smokers" who had stopped smoking since at least last one year. For the current smokers, cigarettes per day also noted. Brief Symptom Inventory, Toronto Alexithymia Scale (TAS), Personal Well-Being Index Adult Form, Positive and Negative Affect Schedule, Experiences in Close Relationships-Revised Scales were used.

Results: $43.6 \%(\mathrm{n}=10,672)$ of the participants were smokers; $5.7 \%$ (1386) were ex-smokers; $50.7 \%(n=12,414)$ were non-smokers. A higher number of daily smoked cigarettes was related to all subscales of Brief Symptom Inventory, TAS - Difficulty in Recognition of emotions, TAS - Difficulty in Expressing Emotions, Positive Affect Score, Negative Affect Score, Avoidance and Anxious Attachment scores $(p<0.05)$. Externally oriented thinking is found to be significantly higher among ex-smokers than current smokers and non-smokers $(p<0.05)$.

Conclusion: The results of the present study indicate that smokers have more psychopathological characteristics in the psychometric evaluation, whereas ex-smokers are found to have similar scores to non-smokers. The higher percentage of externally oriented-thinking style in ex-smokers may suggest that this alexithymic characteristic can help the individual to deal with psychological addiction throughout quitting. On the other hand this result could also be related that stopping smoking leads to greater externally orientated thinking and other changes in psychological characteristics.

Keywords: smoking, quitting, addiction, attachment, alexithymia, well-being

\section{Introduction}

Smoking is one of the outstanding global public health problems. It is also a preventable cause of smoking-related diseases and overall mortality. Currently, there are more than a billion cigarette smokers worldwide, and the number of smokers in developing countries is rising. Tobacco use leads to around 6 million avoidable deaths each year worldwide. ${ }^{1}$ It was reported that two-thirds of smokers wanted to quit, and half of them attempted to quit smoking on their own, but only a small percentage are successful in quitting. ${ }^{2}$

Researchers who examine the risk factors for smoking are often focused on socioeconomic and cultural factors. ${ }^{3}$ In relatively fewer studies focused on psychological mechanisms that pose risk factors for smoking, it was reported that depressive mood, ${ }^{4}$ negative affect, ${ }^{5}$ low levels of self-confidence and personal well-being ${ }^{6}$ were associated
Correspondence: Gökben Hızlı Sayar Üsküdar University, Institute of Socia Sciences, Selmanı Pak Street Altunizade, Istanbul, Turkey

Tel +902164002200

Email gokben.hizlisayar@uskudar.edu.tr 
with smoking addiction. However it is not clear that whether the differing psychological characteristics were present before onset of smoking, whether they are related to uptake or cessation, or whether they are a result of smoking or smoking cessation (or a combination of these). It has been stated that the most successful treatment option in the treatment of smoking addiction is the combined use of pharmacotherapy and psychotherapy. ${ }^{7}$ Another crucial point besides the determination of predisposing psychological factors to smoking addiction would be the definition the common psychological characteristics of those who can successfully stop smoking. Thus, it may be conceivable to increase the success rate of addiction treatments by addressing common psychological traits of ex-smokers.

This study aims to examine the psychological factors associated with smoking and quitting in a broad, nationally representative sample. With the analysis of smoking-related data of TURBAHAR (Addiction Map of Turkey Study) several psychometric characteristics, namely levels of positive and negative emotions, alexithymia, psychiatric symptom levels, and personal well-being, will be compared.

\section{Participants and methods}

This study is a part of Addiction Map of Turkey Study (TURBAHAR), which was carried out throughout Turkey in 2018. The stratified cluster sampling approach based on the NUTS classification (Nomenclature of territorial units for statistics) was used. NUTS is a hierarchical system for dividing up the economic territory of the European Union. ${ }^{8}$ The people residing in 26 NUTS regions of Turkey were included in the study. At least 200 and at most 2000 people were involved in each region. Inclusion criteria for participants were being over 18 years of age and not having a mental illness that prevents the individual from completing questionnaires. Larger samples were selected from the regions with higher densities of population. A total of 24,990 people were interviewed for the study; however, 24,494 individuals met the criteria and completely filled the scales.

Between July 2018 and October 2018, the participants were selected from various fields of study such as schools, municipal buildings, private companies, and public places such as neighborhood units, courses, and charities. Potential participants in the given lists were asked for consent for participating in the study. Individuals aged 18 and over were included in the study. Following the signing of the informed consent form, the questionnaires which were included in the data collection tools were ensured to be filled by the participants based on the self-report of the participant under the supervision of the interviewer. Directives for scales were given both verbally and in written form. The interviews in the study were carried out by clinical psychologists $(n=125)$. Eight hours of training given to ensure consistency in administration of interviews between the 125 psychologists. Any question asked by the participants during the filling in of documents were answered. Filling in the questionnaires took 45 mins on average for each participant. Üsküdar University Ethics Committee of Non-Invasive Researches approved the study.

\section{Instruments}

Sociodemographic Information Form: This form prepared by the researchers includes questions about participants' gender, age, education, marital status, number of children, smoking status, number of daily smoked cigarettes, and history of a lifetime and current psychiatric disorders.

Brief Symptom Inventory (BSI): This instrument was developed by Derogatis; it is a shorter version of the SCL90-R. The instrument consists of 53 items in nine subscales (somatization, obsessive-compulsive disorder, interpersonal sensitivity, depression, anxiety disorders, hostility, phobic anxiety, paranoid ideations, psychoticism), and was adapted to Turkish by Şahin and Durak. ${ }^{9}$ The participants were asked to reply using a five-point scale ranging from "not at all" to "extremely." The alpha coefficients of the factor subscale ranged between 0.70 (for depression) and 0.88 (for somatization). The correlation coefficients of the factor subscale with the other instruments ranged between $-0.45(p<0.001)$ and 0.71 for the Turkish validation study. ${ }^{9}$

Toronto Alexithymia Scale (TAS-20): This scale was developed to investigate alexithymia, defined as not recognizing one's own emotions and excitement. It is a 5 point Likert-type self-report scale consisting of 20 items with three subscales: difficulty identifying feelings (TAS-1), difficulty expressing feelings (TAS-2) and externally oriented thinking (TAS-3). Higher scores indicate higher levels of alexithymia. The scale was developed by Bagby et al. ${ }^{10}$ The Turkish version was developed by Güleç et al. ${ }^{11}$

Personal Well-Being Index Adult Form (PWBI-AF): Personal well-being index, subjective well-being developed by International Wellbeing Group in 2006, 11-point (0-10) Likert type is a measurement tool which aims to measure the satisfaction levels of eight areas in the life of individuals in accordance with the structure of the concept. ${ }^{12}$ These eight areas were sorted as: quality of life; individual health; 
success in life; bilateral relations; personal security; social belonging; looking to the future with confidence; and spirituality. The PWBI-AF, which was adapted by Meral to Turkish, consisted of 8 items, all of which are positive; and the scale can be graded with a maximum score of 80 in the scale. ${ }^{13}$ The Cronbach Alpha internal consistency coefficient of the scale for this research is calculated as 89 .

Positive and Negative Affect Schedule (PANAS): The PANAS is a 20 -item self-report measure of positive and negative affect at a given point in time using a 5 point Likert scale. ${ }^{14}$ Participants respond to 20 adjectives describing affect. Gençöz conducted its validity and reliability for Turkish. ${ }^{15}$ The Turkish version of PANAS has demonstrated good internal consistency (0.83-0.86) and moderate concurrent validity $(0.40-0.54){ }^{15}$

Experiences in Close Relationships-Revised (ECR-R): The validity and reliability of the scale developed by Fraley and Shaver, was carried out by Selçuk and his friends in $2005 .{ }^{16}$ There are a total of 36 items in seven-item Likert: 18 in anxiety and 18 in avoidance subscales. The score from each subscale varies between 18 and 126, and the higher scores are related to avoidant attachment or anxious attachment styles. While the Cronbach alpha coefficient of the avoidance sub-dimension is 0.90 , the Cronbach alpha coefficient of the anxiety sub-dimension is 0.86 . Test-retest reliability coefficients for avoidance and anxiety dimensions of the scale are 0,81 and 0,82 , respectively. ${ }^{17}$

The data of the study were analyzed using SPSS-21. Sociodemographic characteristics and data from 5 different scales were included in the analysis. Participants were divided into three groups as smokers, non-smokers, and "ex-smokers" who had stopped smoking for at least last one year. A categorical variable for the number of cigarettes smoked daily was created with the following groups: "1-10 cigarettes", "11-20 cigarettes", "21-40 cigarettes", "41 and more cigarettes". One-way ANOVA with Tukey post-hoc tests was used to determine significant differences among current smoking status groups and among the number of cigarettes per day groups.

\section{Results}

In this study, $49.8 \%(n=12,191)$ of the participants were female and $50.2 \%(n=12,303)$ were male. The age range of the participants was $18-81$, and the mean age was 32.3 \pm 11.06 for females and $31.22 \pm 10.7$ for males. $43.1 \%$ of the participants are married, and $39.3 \%$ have children. $43.6 \%$ $(n=10,672)$ of the participants were smokers; $5.7 \%(1386)$ were ex-smokers; $50.7 \%(\mathrm{n}=12,414)$ were non-smokers.
Of the male respondents, $52.9 \%$ were smokers, $40.1 \%$ were non-smokers, and $6.9 \%$ were ex-smokers. Of the female respondents, $34.1 \%$ were smokers, $61.3 \%$ were non-smokers, and $4.4 \%$ were ex-smokers. The percentage of those who successfully quit smoking was $11.5 \%$ in male smokers and $11.45 \%$ in female smokers (ratio of exsmokers to ex-smokers + current smokers). There are no statistically significant differences observed between the percenteges of quitting smoking considering the gender.

It was noticed that the increase in the number of daily smoked cigarettes was related to all subscales of BSI, TAS Difficulty in Recognition of emotions, TAS - Difficulty in Expressing Emotions, Positive Affect Score, Negative Affect Score, ECR-R - Avoidance and Anxious Attachment scores $(p<0.01)$. The increase in the number of cigarettes smoked daily was found to be related to lower Personal Well-being scores $(p<0.01)$. Relationship of scores of scales with number of daily smoked cigarettes is given in Table 1 .

Smokers were found to have a statistically significant difference in TAS-Difficulty in Identifying Emotions, Positive Affect, Negative Affect, and Anxious Attachment, all sub-scales of BSI when compared to nonsmokers and ex-smokers $(p<0.01)$. Scores of ex-smokers resembled considerably to those of non-smokers compared to non-smokers. However, the ex-smokers had significantly higher Brief Symptom Inventory scored compared to non-smokers $(p<0.01)$. Ex-smokers were also less likely to have avoidant attachment characteristics and have higher scores of externally oriented thinking compared to smokers and non-smokers, and statistically significant differences were found between groups $(p<0.01)$. The personal well-being of non-smokers was found to be higher than that of smokers, and ex-smokers $(p<0.05)$. Relationship of scores of scales with smoking status is given in Table 2.

\section{Discussion}

This is the most extensive study ( $\mathrm{n}=24.494)$ conducted in Turkey that focused on psychometric data related to smoking and quitting. The results reveal that the percentage of active smoking is $54.9 \%$ for men and $34.1 \%$ for women. In Turkish Statistical Institute data, the percentage of smoking in 2012 was $41.4 \%$ for men and $13.1 \%$ for women in Turkey. ${ }^{18}$ The fact that the present study (TURBAHAR) included only people aged 18 and over, could have built this difference. Turkish Statistical Institute data with under 18 years of age excluded, gives the similar percentages for smokers. However, rates of successfully quitting were found to be equal $(11.5 \%)$ for 


\begin{tabular}{|c|c|c|c|c|c|}
\hline & оे & ठै. & оे & \% & 容 \\
\hline & 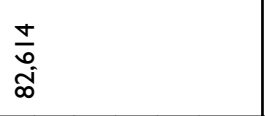 & $\frac{8}{\infty 0^{\circ}}$ & $\begin{array}{l}\infty \\
\infty \\
\infty \\
\infty \\
\infty\end{array}$ & $\stackrel{\stackrel{0}{\leftarrow}}{\stackrel{\pi}{\pi}}$ & $\begin{array}{l}\stackrel{0}{m} \\
\text { ồ } \\
\underline{n}\end{array}$ \\
\hline & 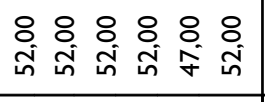 & 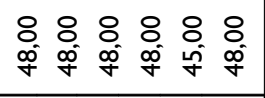 & 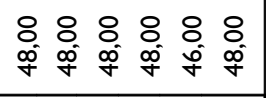 & 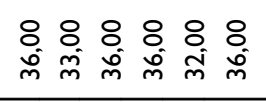 & 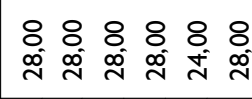 \\
\hline & 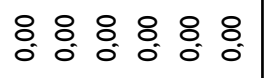 & 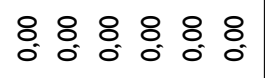 & : : $: 0$. & : : : : : : : : & : $8: 80.8$ \\
\hline & 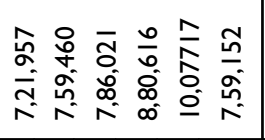 & 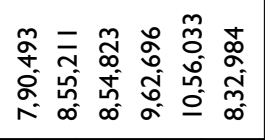 & 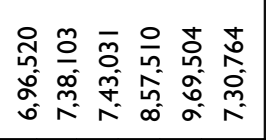 & 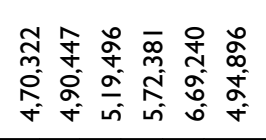 & 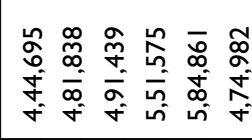 \\
\hline & 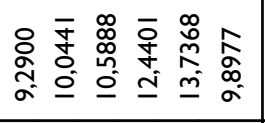 & 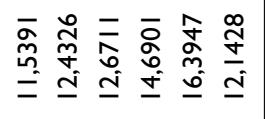 & 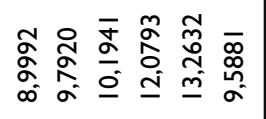 & 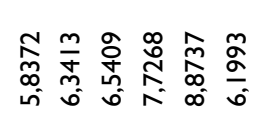 & 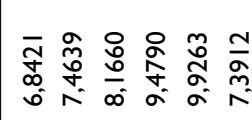 \\
\hline & 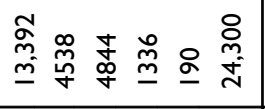 & 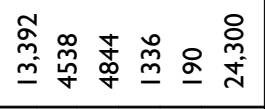 & 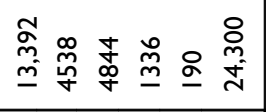 & 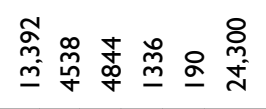 & 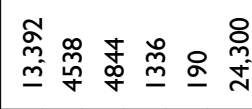 \\
\hline & 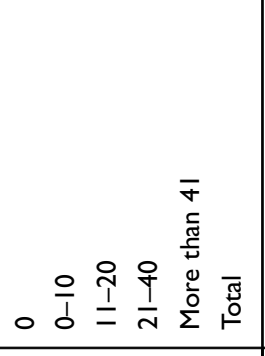 & 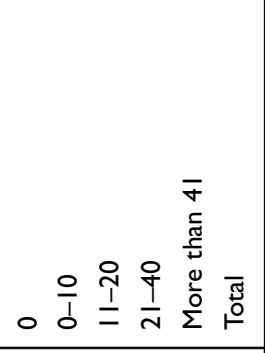 & 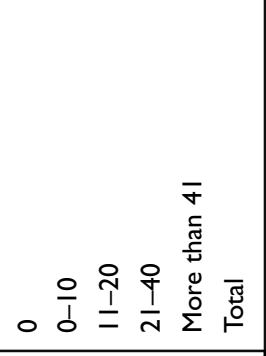 & 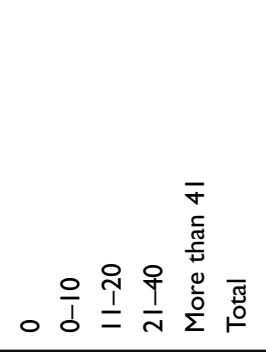 & 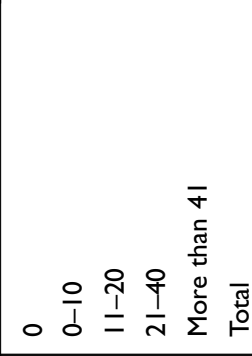 \\
\hline & 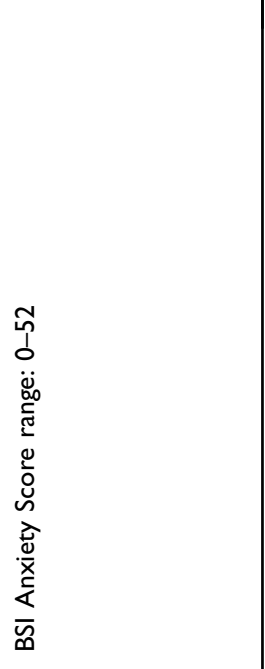 & 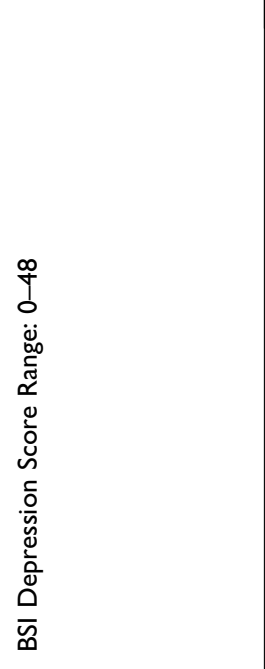 & 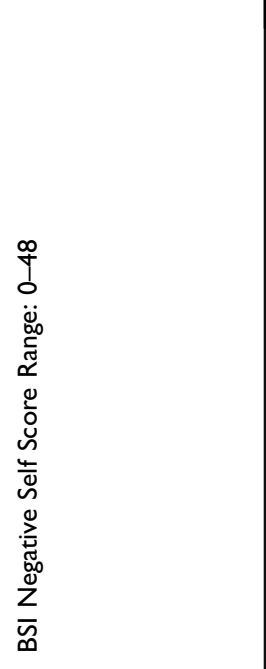 & 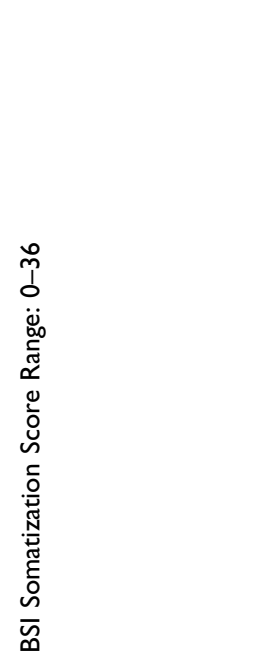 & 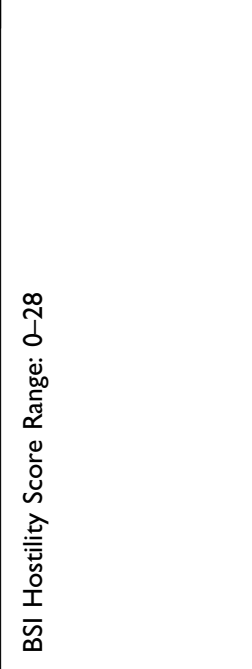 \\
\hline
\end{tabular}




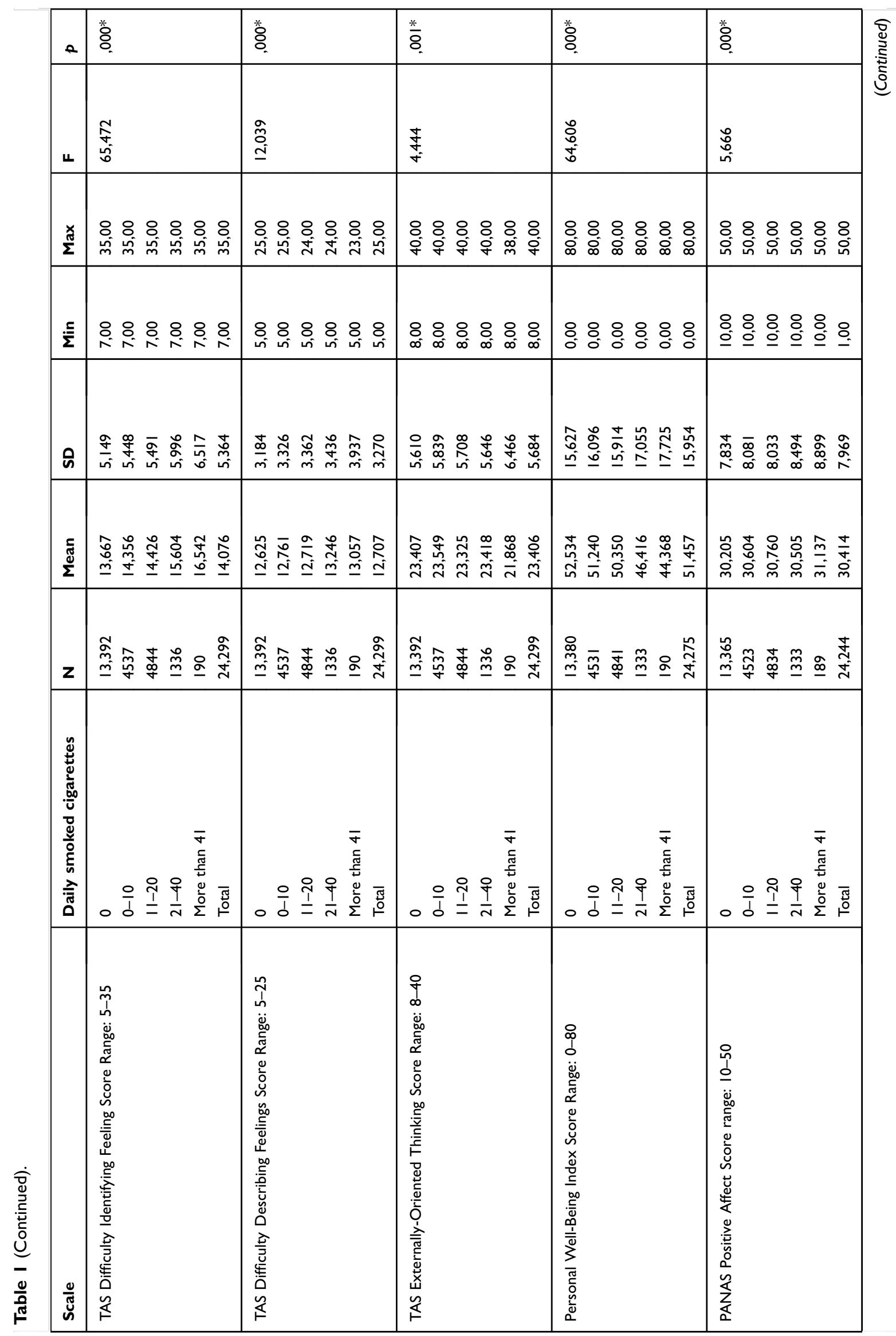




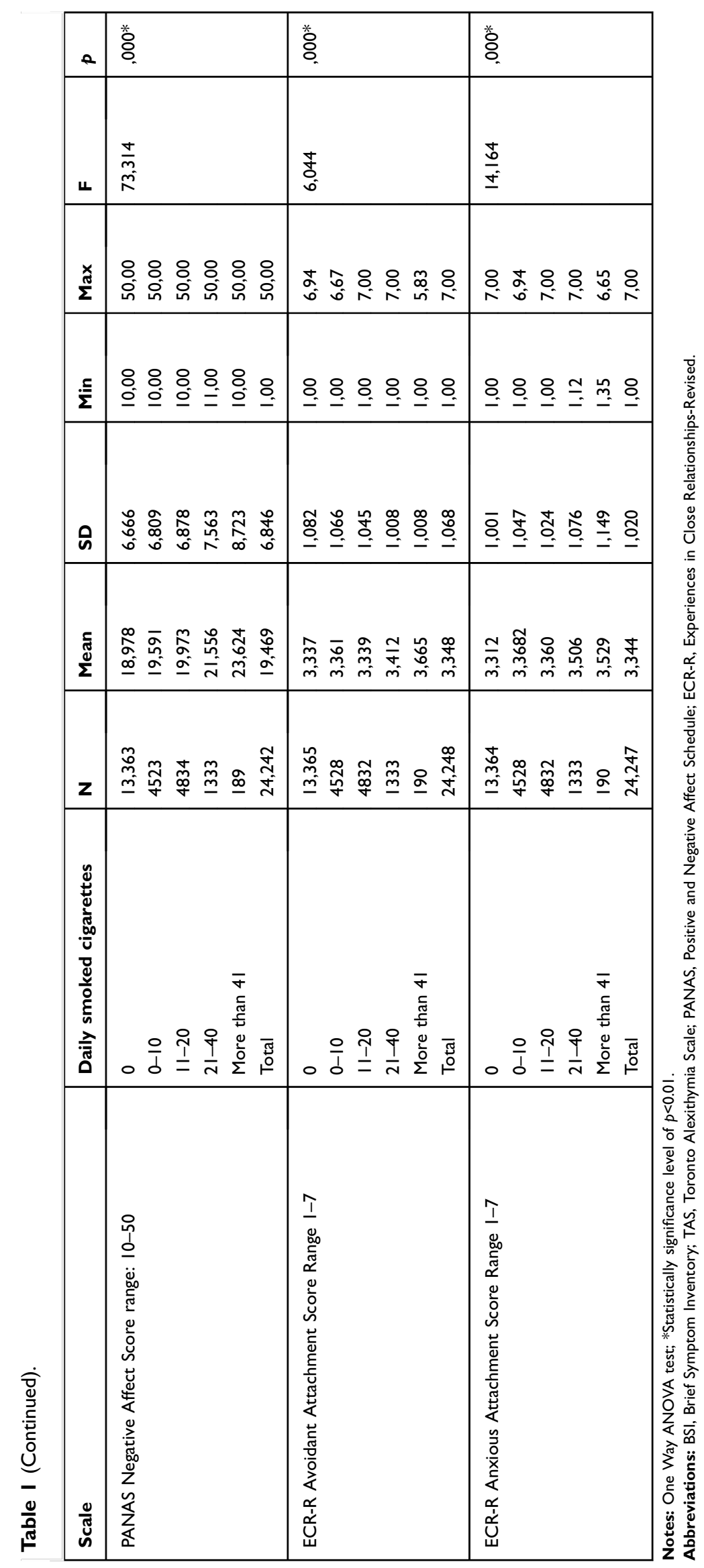




\begin{tabular}{|c|c|c|c|c|c|c|c|c|}
\hline & $\underline{\Lambda} \underline{\Lambda}$ & $\Lambda \underline{\Lambda}$ & $\Lambda \underline{\Lambda}$ & $\Lambda \underline{\Lambda}$ & $\begin{array}{l}\hat{\hat{n}} \\
\underline{\Lambda}\end{array}$ & $\Lambda \underline{\Lambda}$ & $\underline{\Lambda}$ & $\overline{\hat{m}} \hat{m}$ \\
\hline a & \% & ठैं & ӧ & \%े & оे & 容 & ठै. & $\frac{*}{8}$ \\
\hline 4 & $\begin{array}{l}\text { N } \\
\text { 定 } \\
=\end{array}$ & $\frac{\frac{a}{m}}{a^{2}}$ & $\begin{array}{l}\stackrel{\overline{0}}{\underline{\Xi}} \\
=\end{array}$ & 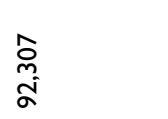 & 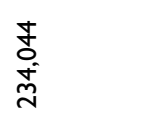 & \begin{tabular}{|l}
$\infty$ \\
$\stackrel{\infty}{2}$ \\
$\alpha^{\infty}$
\end{tabular} & 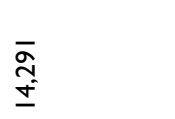 & 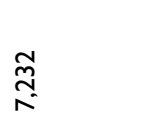 \\
\hline 齐 & $\begin{array}{l}8 \text { 8 } 8 \text { : } \\
\text { î } \\
\text { ñ }\end{array}$ & 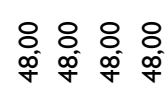 & 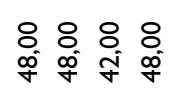 & 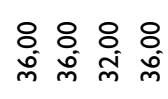 & 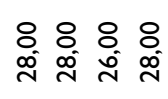 & $\begin{array}{l}88 \\
8 \\
m \\
m\end{array}$ & 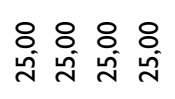 & 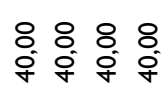 \\
\hline$\frac{\Xi}{\Sigma}$ & $\begin{array}{llll}8 & 8 & 8 & 8 \\
\circ & 0 & 0 & 0\end{array}$ & $\begin{array}{llll}8 & 8 & 8 \\
\circ & 0 & 0 & 0\end{array}$ & $\begin{array}{llll}8 & 8 & 8 & 8 \\
0 & 0 & 0 & 0\end{array}$ & $\begin{array}{llll}8 & 8 & 8 \\
\circ & 0 & 0 & 0\end{array}$ & $\begin{array}{llll}8 & 8 & 8 \\
0 & 0 & 0\end{array}$ & 윳 : & $\begin{array}{l}8 \\
\text { in }\end{array}$ & $\begin{array}{cccc}8 & 8 & 8 & 8 \\
\infty & \infty & 8 \\
\infty & \infty & \infty \\
\infty\end{array}$ \\
\hline 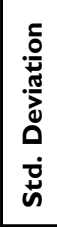 & 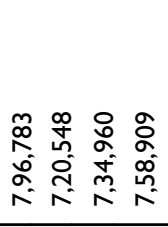 & 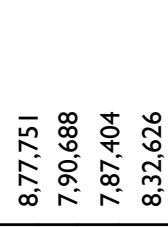 & 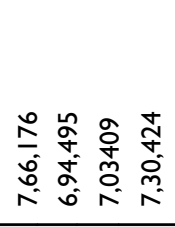 & 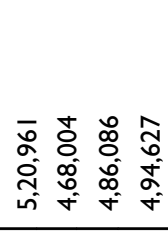 & 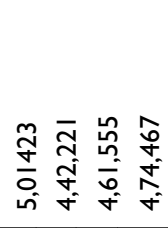 & 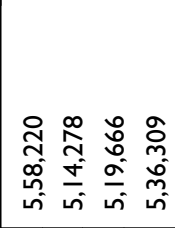 & 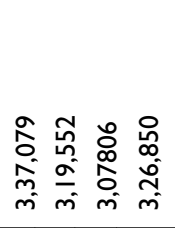 & 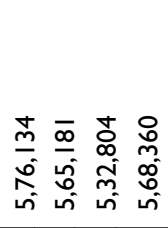 \\
\hline$\frac{\tilde{\sigma}}{\Sigma}$ & 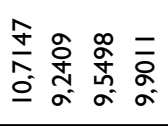 & 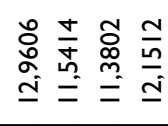 & 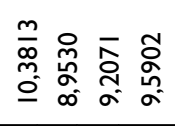 & 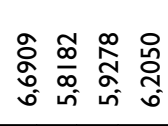 & 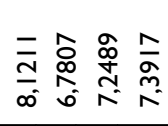 & 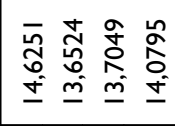 & 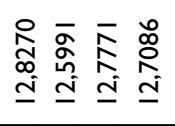 & 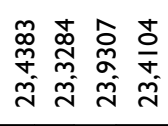 \\
\hline $\mathbf{z}$ & 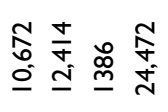 & 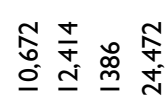 & 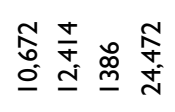 & 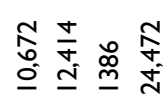 & 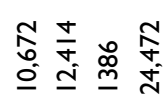 & 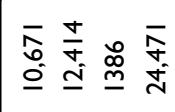 & 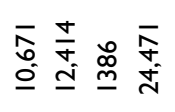 & 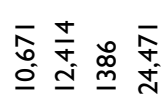 \\
\hline & 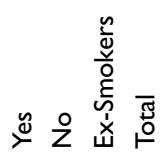 & 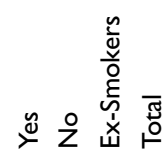 & 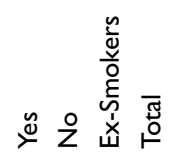 & ¿ & ֻ & 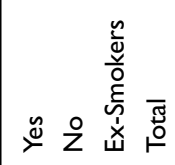 & 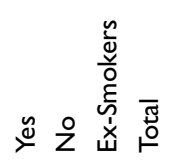 & 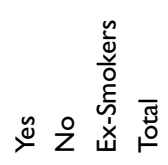 \\
\hline & 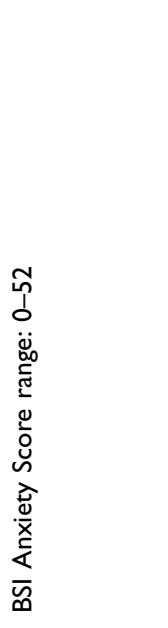 & 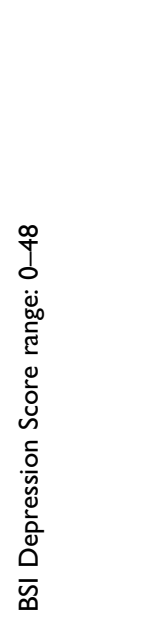 & 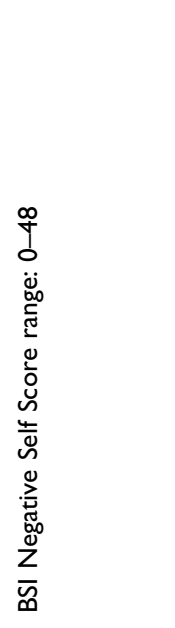 & 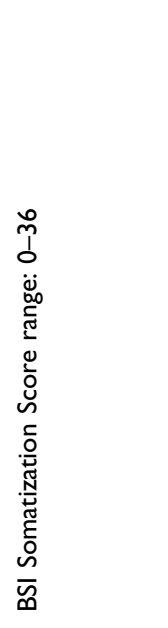 & 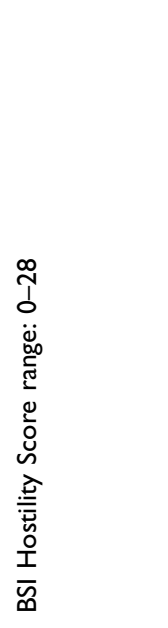 & 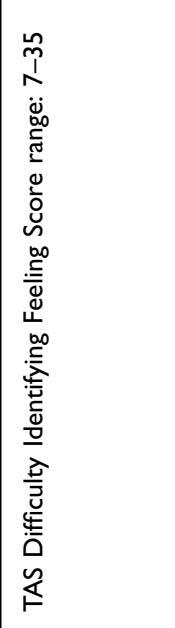 & 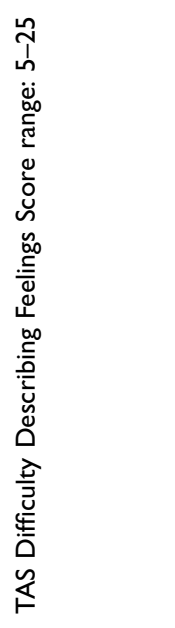 & 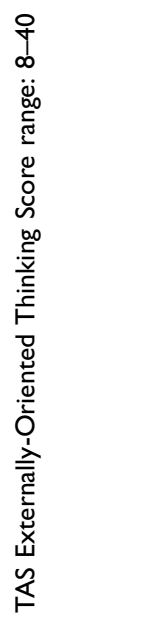 \\
\hline
\end{tabular}




\begin{tabular}{|c|c|c|c|c|c|}
\hline & $\overline{\hat{N}} \hat{\sim}$ & $\Lambda \underline{\Lambda}$ & $\Lambda \Lambda$ & $\overline{\mathrm{v}} \underset{\mathrm{v}}{\mathrm{N}}$ & $\underline{\Lambda} \underline{\Lambda}$ \\
\hline a & \% & 。․ & \% & \% & 。ั้ \\
\hline L & $\begin{array}{c}\infty \\
\substack{0 \\
\infty} \\
\infty\end{array}$ & $\begin{array}{l}\overline{\check{\alpha}} \\
\dot{ \pm}\end{array}$ & $\frac{\stackrel{\rho}{m}}{\infty}$ & 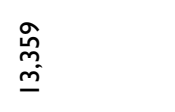 & $\begin{array}{l}\stackrel{8}{\infty} \\
\infty \\
\infty\end{array}$ \\
\hline$\underset{\Sigma}{\frac{x}{\Sigma}}$ & 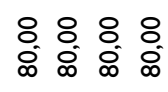 & 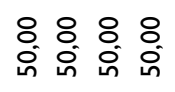 & 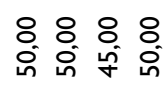 & 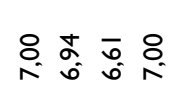 & 咨 : \\
\hline$\frac{\Xi}{\Sigma}$ & : : $: 0$ & 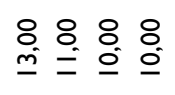 & $\begin{array}{l}8: 8 \\
= \\
=\end{array}$ & 8.80 & 8.8. \\
\hline 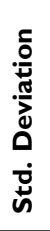 & 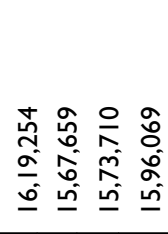 & 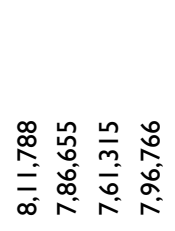 & 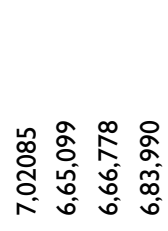 & 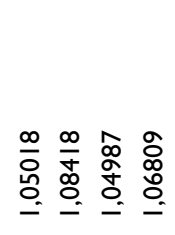 & 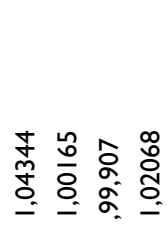 \\
\hline 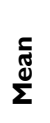 & 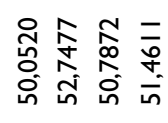 & 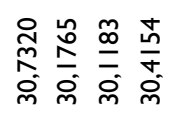 & 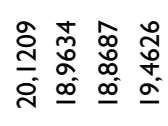 & 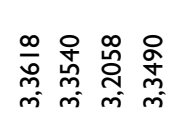 & 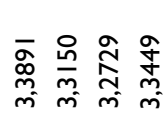 \\
\hline $\mathbf{z}$ & 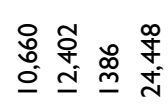 & 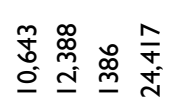 & 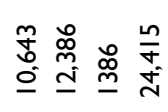 & 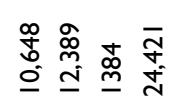 & 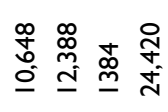 \\
\hline & ๗ & 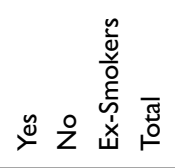 & 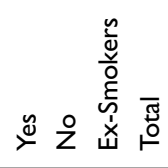 & 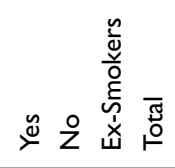 & ֻ \\
\hline & 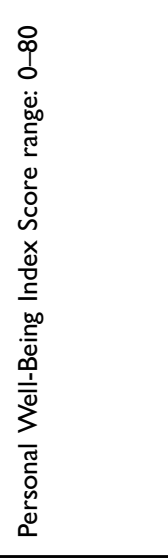 & 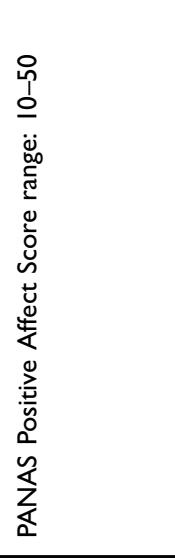 & 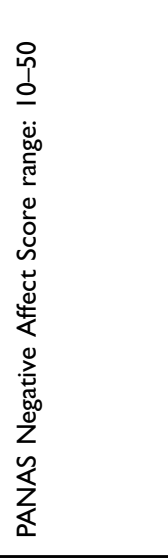 & 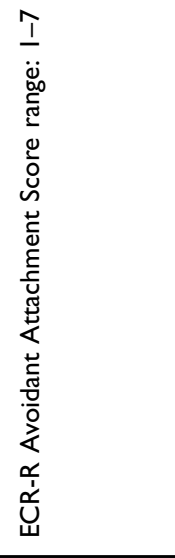 & 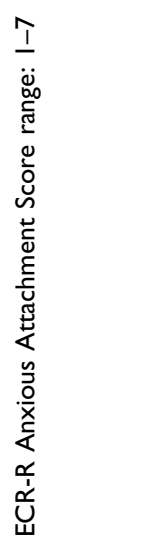 \\
\hline
\end{tabular}


both genders. Also, the data revealed that the characteristics of ex-smokers are substantially comparable to those of non-smokers. Although the smoking rates of males were higher than that of females, successfully quitting smoking was found to be gender-independent.

Several scales were used in the present study to evaluate the relationship between smoking and psychological variables. The results indicate that a higher daily number of cigarettes is related to increased anxious and avoidant attachment styles. The high prevalence of insecure attachment (both anxious and avoidant attachment styles) may have a significant impact on smoking and quitting smoking rates (approximately $40 \%$ of the sample population had high attachment insecurity) and may contribute to nicotine addiction. It was reported that an insecure attachment is associated with adult psychopathology, including mood, anxiety, and behavioral disorders. ${ }^{19,20}$ In the present study, the avoidant attachment styles were observed at a lower percentage in exsmokers compared to current smokers and non-smokers. In the literature, individuals with avoidant attachment style were reported as having a higher physiological reaction to negative emotions. ${ }^{21,22}$ It can be hypothesized that being more sensitive to negative emotions makes the individuals with avoidant attachment styles less successful in quitting smoking. Individuals with anxious attachment styles may be in the higher risk group to start smoking; however, they can deal better with quitting due to their lower avoidant attachment characteristics.

One of the remarkable results of the present study is that both positive and negative emotions scaled by PANAS are higher in smokers compared to ex-smokers and nonsmokers. There is some evidence that PANAS could predict the response to nicotine therapy. ${ }^{23}$ In their study, Veselka et al reported that the smoking behavior of young people is related to self-efficacy and negative emotions. ${ }^{24}$ Evidence suggests that high levels of negative emotions (depression, anxiety, and anger) is associated with smoking behavior. ${ }^{25,26}$ Several recent studies showed the relationship between negative emotion and smoking addiction. ${ }^{27,28}$ On the other hand, positive emotion was found to be associated with smoking desire in smokers. ${ }^{29}$

In the present study, BSI was found to be high in all subscales among current smokers. Dimitrios et al, in a study compared psychopathological features of smokers and non-smokers, used the SCL-90 scale. Their results also indicate that smokers have higher scores in depression, interpersonal sensitivity, hostility, somatization compared to non-smokers. ${ }^{30}$ Several previous studies found anxiety, ${ }^{31}$ hostility, ${ }^{32}$ depression ${ }^{32-34}$ and somatization ${ }^{32}$ characteristics related with smoking. Cross-sectional studies have consistently found that smokers have a higher level of hostility than non-smokers. ${ }^{35}$ Previous researches indicate that among non-smokers, smoking initiation was predicted prospectively by higher extraversion, and among smokers, smoking cessation was negatively associated with neuroticism. ${ }^{36}$

One of the remarkable findings of the present study is that externally oriented thinking is found to be significantly higher among ex-smokers than current smokers and nonsmokers. Alexithymia is a multidimensional concept that associates an emotional component, focused on the difficulty in identifying and describing feelings, with a cognitive one, centered on the use of a concrete and poorly introspective way of thinking. TAS-20 tests three dimensions of alexithymia: difficulty in recognizing emotions, difficulty in defining emotions, and externally oriented thinking. While the first two dimensions point to emotional awareness, externally oriented thinking refers to a cognitive tendency to focus on surface themes rather than psychological experiences in order to prevent affect. In pathological states, these mechanisms may over-protect a person from stress reactivity to any challenge, which promotes the subject's successful coping with negative experience. ${ }^{37}$ It has been reported that externally oriented-thinking is associated with low sensitivity to emotional separation. ${ }^{38}$ The higher percentage of externally oriented-thinking style in ex-smokers suggests that this alexithymic characteristic may help the individual to deal with psychological addiction throughout quitting. In general, classical psychotherapeutic approaches often try to determine the internal causes of a problem and they use techniques to raise awareness that physical symptoms may be related to stressful situations in individuals with externally oriented-thinking styles. However, the findings of our study suggest that externally oriented affect may be an advantage for the individual throughout the process of quitting. Therefore, it is recommended to review the techniques used in psychotherapies of addiction.

This report has several limitations. First, the results are gathered from observational data to draw causal conclusions, and the assuming that the psychological characteristics identified lead to smoking status, however these characteristics might be a result of smoking or smoking cessation. Second, the smoking status was self-reported and not validated by biochemical testing. The research was conducted only in cities, so results are not generalizable to rural population. 


\section{Conclusion}

In conclusion, the results of the present study indicate that smokers have more psychopathological characteristics in the psychometric evaluation, whereas ex-smokers are found to have similar scores to non-smokers. The findings suggest that high externally oriented thinking style and low anxious attachment style characteristics are associated with successful quitting. The externally oriented thinking style may help the quitter by giving emotional or cognitive detachment from the negative content processing related to quitting.

Targeted interventions can accelerate progress toward reducing adult smoking prevalence. Motivational information to a patient is more effective if it is personally relevant to a patient. The health care providers must encourage the patient to identify potential benefits of quitting smoking such as improved physical and mental health. The common purpose of many medical methods used to help smoking cessation is to overcome the psychological dependence and physical dependence of smoking in individuals. Further clinical trials with carefully monitored interventions to determine the effect of externally oriented thinking on success of smoking cessation are needed.

\section{Ethics approval and informed consent}

The research project and the terms of informed consent for participants were analyzed and approved by the Üsküdar University Ethics Committee of Noninvasive Studies according to protocol no B.08.6.YÖK.2.ÜS.0.05.0.06/ $2018 / 800$

\section{Acknowledgments}

The authors would like to acknowledge Prof. Dr. Nevzat Tarhan and Tayfun Gözler for their support and assistance with this project. The authors also would like to acknowledge A. Yadigar Terzi, Mübarek Aydın, Beyza Arıduru, Şura Yılmaz, Rukiye Aydın, Gizem Zorlu, Işıl Toka, Koray Acar, Seher Tunçer, Sedef Ceylan Çevik, Beste Kara, Ekin Alan, B. Naz Alır, Esra Elitaş, Cemre Menteşe, Deniz Dirin, Gaye Göklü, İlayda Sezen, Sena Kuyumcuoğlu, Sude Yaşa, Nida Boğa, Melike Çapkın, Berna Baydil, Sena Çalın, Rüveyda Ata, Narin Özer, Şebnem Geylani, D.Derya Sarı, Metehan Şenocak, Berat Sena Varlık, Okan Dursun, Enise Öziç, Nuray Yılmaz, Cansu Yılmaz, Samet Çoban, Şeyda Ak, Kübra Mete, Zehra Aydınol, Şenay Dayan, Merve Akçıl, Mihriban Taşpınar, Hülya Aydın, Elif Kocaman, H.Su Kökçü,
Özge Engin, Büşra Sürmeli, N. Dilayra Ayvalığlu, Ö. Sena Altaş, Yusuf Yerli, Zeynep Kahveci, Ali Işıtaş, Betül Aktürk, Kübra Bakırcı, Sezer Akşit, Beyza Yaz, Berika Gizem Yıldırım, Taner Güler, Zeynep Kır, Kübra Tehci, Berfin Demir, Burcu Beyler, Canan Bulduk, Gülsüm Bircan, E. Çağla Zengül, Aslı Korkmaz, Zeliha Şahin, Aylin Furun, Ezgi Akyel, E.Damla Erol, Hilal Teberoğlu, Aybüke Karataş, Ege Kayhan, Gülçin Mete, Kübra Yılmaz, Merve Beşik, C. Hazal Çevik, Haydar Keşen, Rabia Üçeş, Seda Bay, Tuğba Bakar, Hasretnur Enginyurt, H. Mehveş Onar, Serap Yüksel, Ş. Hazal Kaya, Büşra Keyik, Gizem Öztürk, Mustafa Günel, Zeynep Aygün, Mine Sütcü, Şahika Tural, Z. Büşra Dursun, A. Gül Akbaba, Beyza Yüksel, Burak Sunkur, Rıdvan Yamangil, B.Nilay Şahin, Hülya Özgür, Büşranur Keleş, Derya Zaimoğlu, A.N. Hazal Sezen, N. Handan Macit, Büşra Boz, Hatice Yıldız, Sedef Evlice, Yağmur Yazar, Melisa Er, Bedriye Ege, Osman İcil, Merve Gören, Merve Erten, Nurcan Sönmez, Tuğçe Bilgin, Berna Tefenlili, Cemile Sargın, İclal G. Atayay, Sena Sarıtabak, Ayşenur Yılmaz, Feyza Sümbül, İ. Büşra Ayhan, İ. Nur Ülkü, Ayşegül Özkula, Burak İyidir, Merve Paçac1, Mülkiye Özden and Özge Bedir for assistance with data collection for this research. This work was supported by Üsküdar University.

\section{Author contributions}

All authors contributed toward data analysis, drafting and critically revising the paper, gave final approval of the version to be published, and agreed to be accountable for all aspects of the work.

\section{Disclosure}

The authors report no conflicts of interest in this work.

\section{References}

1. World Health Organization. WHO report on the global tobacco epidemic, 2011. Warning about the dangers of tobacco [Internet]; 2011 [cited November 26, 2018]. Available from: https://www.who.int/ tobacco/global_report/2011/en/

2. American Cancer Society. Why people start using tobacco, and why it is hard to stop; 2015. Available from: https://www.cancer.org/cancer/ cancer-causes/tobacco-and-cancer/why-people-start-using-tobacco. html

3. Twyman L, Bonevski B, Paul C, Bryant J. Perceived barriers to smoking cessation in selected vulnerable groups: a systematic review of the qualitative and quantitative literature. BMJ Open. Brit Med J Publ Group. 2014;4(12):1-15.

4. Thorberg FA, Lyvers M. Negative Mood Regulation (NMR) expectancies, mood, and affect intensity among clients in substance disorder treatment facilities. Addict Behav. 2006;31(5):811-820. doi:10.1016/j. addbeh.2006.01.008 
5. Baker TB, Piper ME, McCarthy DE, Majeskie MR, Fiore MC. Addiction motivation reformulated: an affective processing model of negative reinforcement. Psychol Rev. 2004;111(1):33-51. doi:10.1037/0033-295X.111.1.33

6. Habibi M, Hosseini F, Darharaj M, Moghadamzadeh A, Radfar F, Ghaffari Y. Attachment style, perceived loneliness, and psychological well-being in smoking and non-smoking university students. J Psychol Int Appl. 2018;152(4):226-236. doi:10.1080/00223980. 2018.1446894

7. Ingersoll KS, Cohen J. Combination treatment for nicotine dependence: state of the science. Subst Use Misuse. 2005;40(13-14):1923-1943. doi:10.1080/10826080500294817

8. European Commission - Eurostat NUTS - Nomenclature of territorial units for statistics. Available from: https://ec.europa.eu/eurostat/web/ nuts/statistical-regions-outside-eu. Accessed December 20, 2018.

9. Şahin NH, Durak AB. Kısa Semptom Envanteri: türk gençleri için uyarlanması (Brief Symptom Inventory: adaptation for Turkish youth- In Turkish). Turk Psikoloji Dergisi. 1994;9(31):44-56.

10. Bagby RM, Taylor GJ, Parker JDA. The twenty-item Toronto Alexithymia scale-II. Convergent, discriminant, and concurrent validity. J Psychosom Res. 1994;38(1):33-40. doi:10.1016/00223999(94)90006-X

11. Gulec H, Kose S, Güleç MY, et al. Reliability and factorial validity of the Turkish version of the 20-item Toronto alexithymia scale (TAS-20). Klinik Psikofarmakoloji Bulteni. 2009;19(3):215-221.

12. International Wellbeing Group. Personal Wellbeing Index-Adult. Melbourne: Australian Centre on Quality of Life, Deakin University. [cited November 26, 2018]. Available from: http://www. acqol.com.au/uploads/pwi-a/pwi-a-english.pdf

13. Meral BF. Kişisel iyi oluş indeksi-yetişkin Türkçe formunun psikometrik özellikleri. (Personal well-being index - psychometric properties of adult Turkish form - in Turkish). J Happiness Well-Being. 2014;2(2):119-131.

14. Watson D, Clark LA, Tellegen A. Development and validation of brief measures of positive and negative affect: the PANAS scales. J Pers Soc Psychol. 1988;54(6):1063-1070. doi:10.1037/0022-3514.54.6.1063

15. Gençöz T. Pozitif ve Negatif Duygu Ölçeği: geçerlik ve güvenirlik çalışması (Positive and Negative Emotion Scale: validity and reliability study - in Turkish). Turk Psikoloji Dergisi. 2000;15(46):19-26.

16. Fraley RC, Waller NG, Brennan KA. An item response theory analysis of self-report measures of adult attachment. J Pers Soc Psychol. 2000;78(2):350-364. doi:10.1037/0022-3514.78.2.350

17. Selcuk E, Gunaydın G, Sümer N, Uysal A. Yetişkin bağlanma boyutları için yeni bir ölçüm: yakın İlișkilerde Yaşantılar Envanteri-II'nin Türk örnekleminde psikometrik açıdan değerlendirilmesi (A new measurement for adult attachment dimensions: psychometric evaluation of Turkish version of Experiences in Close Relationships-II in Turkish sample- In Turkish). Turk Psikoloji Yazlları. 2005;8(16):1-11.

18. Turkish Statistical Institute. Global Adult Tobacco Survey [Internet]. [cited January 29, 2019]. Available from: http://www.tuik.gov.tr/ PreHaberBultenleri.do?id=13142. Accessed 4 July 2019.

19. Bakermans-Kranenburg M, van IJzendoorn MH. The first 10,000 Adult Attachment Interviews: distributions of adult attachment representations in clinical and non-clinical groups. Attach Hum Dev. 2009;11(3):223-263. doi:10.1080/14616730902814762

20. Kassel JD, Wardle M, Roberts JE. Adult attachment security and college student substance use. Addict Behav. 2007;32(6):1164-1176. doi:10.1016/j.addbeh.2006.11.012

21. Feeney BC, Kirkpatrick LA. Effects of adult attachment and presence of romantic partners on physiological responses to stress. J Pers Soc Psychol. 1996;70(2):255-270. doi:10.1037/0022-3514.70.2.255
22. Dozier M, Kobak RR. Psychophysiology in attachment interviews: converging evidence for deactivating strategies. Child Dev. 1992;63 (6):1473-1480. doi:10.2307/1131569

23. Lerman C, Roth D, Kaufmann V, et al. Mediating mechanisms for the impact of bupropion in smoking cessation treatment. Drug Alcohol Depend. 2002;67(2):219-223. doi:10.1016/S0376-8716(02)00067-4

24. Veselska Z, Madarasova Geckova A, Reijneveld SA, Van Dijk JP. Self-efficacy, affectivity and smoking behavior in adolescence. Eur Addict Res. 2011;17(4):172-177. doi:10.1159/000326071

25. Gaudreau P, Sanchez X, Blondin JP. Positive and negative affective states in a performance-related setting testing the factorial structure of the PANAS across two samples of French-Canadian participants. Eur J Psychol Assess. 2006;22(4):240-249. doi:10.1027/1015-5759. 22.4 .240

26. Scott Killgore WD. Evidence for a third factor on the positive and negative affect schedule in a college student sample. Percept Mot Skills. 2000;90(1):147-152. doi:10.2466/pms.2000.90.1.147

27. Conklin CA, Perkins KA. Subjective and reinforcing effects of smoking during negative mood induction. J Abnorm Psychol. 2005;114 (1):153-164. doi:10.1037/0021-843X.114.1.153

28. Vinci C, Copeland AL, Carrigan MH. Exposure to negative affect cues and urge to smoke. Exp Clin Psychopharmacol. 2012;20 (1):47-55. doi:10.1037/a0025267

29. Vinci C, Kinsaul J, Carrigan MH, Copeland AL. The relationship between smoking motives and smoking urges experienced in response to a negative affect induction. Addict Behav. 2015;40:96-101. doi:10.1016/j.addbeh.2014.09.007

30. Dimitrios G, Mamplekou E, Dimitriadis PG, Dimitriadis GD, Papageorgiou C. The association between smoking and psychopathology adjusted for body mass index and gender. Australas Psychiatry. 2016;24(5):441-444. doi:10.1177/1039856216646228

31. Rondina RDC, Martins RA, Manzato AJ, Refberg B, Botelho C. Comparative study between the characteristics of the repertoire of social skills from smokers and ex-smokers. Interamerican J Psychol. 2015;48(3):308-314

32. Mousavi AS, Matinkhah F, Maadani MR, Masjedi MR. Psychological problems and cigarette smoking in Tehran University students in 2010. Tanaffos. 2012;11(3):42-48.

33. Rondina RDC, Gorayeb R, Botelho C. Psychological characteristics associated with tobacco smoking behavior. Jornal Brasileiro De Pneumologia. 2007;33(5):592-601. doi:10.1590/S1806-3713200700 0500016

34. Bunde J, Suls J. A quantitative analysis of the relationship between the Cook-Medley Hostility Scale and traditional coronary artery disease risk factors. Health Psychol. 2006;25(4):493-500. doi:10.1037/ 0278-6133.25.4.493

35. Hakulinen C, Hintsanen M, Munafò MR, et al. Personality and smoking: individual-participant meta-analysis of nine cohort studies. Addiction. 2015;110(11):1844-1852. doi:10.1111/add.13079

36. Luminet O, Rimé B, Bagby RM, Taylor GJ. A multimodal investigation of emotional responding in alexithymia. Cogn Emot. 2004;18 (6):741-766. doi:10.1080/02699930341000275

37. Davydov DM, Zech E, Luminet O. Affective context of sadness and physiological response patterns. J Psychophysiol. 2011;25:67-80. doi:10.1027/0269-8803/a000031

38. Davydov DM, Luminet O, Zech E. An externally oriented style of thinking as a moderator of responses to affective films in women. Int J Psychophysiol. 2013;87(2):152-164. doi:10.1016/j.ijpsycho. 2012.12.003 


\section{Publish your work in this journal}

Neuropsychiatric Disease and Treatment is an international, peerreviewed journal of clinical therapeutics and pharmacology focusing on concise rapid reporting of clinical or pre-clinical studies on a range of neuropsychiatric and neurological disorders. This journal is indexed on PubMed Central, the 'PsycINFO' database and CAS, and is the official journal of The International Neuropsychiatric Association (INA). The manuscript management system is completely online and includes a very quick and fair peer-review system, which is all easy to use. Visit http://www.dovepress.com/testimonials.php to read real quotes from published authors. 